\title{
Impact of lifestyle in middle-aged women on mortality: one article and many questions
}

The Royal College of General Practitioner's (RCGP's) Oral Contraception Study was set up to explore the long-term health effects of oral contraception. ${ }^{1}$ The recruitment of 47000 women by 1400 GPs in the late 1960s was a monumental achievement. Not only has the Oral Contraception Study achieved its original objectives but, given the size of the cohort, it has proven to be a valuable resource to study many diverse issues, including the prevalence of chronic pain and the relationship of tubal sterilisation and subsequent allcause death.

By 1994 a quarter of the women from the original cohort were still under observation and were invited to complete a questionnaire which included questions on their current health status and lifestyle. With a response rate of $85 \%$ this cross-sectional survey, combined with baseline data from the cohort study, has enabled Iversen and colleagues ${ }^{2}$ to investigate the relationship between combinations of risk factors and all cause mortality in women. The study, published in this month's edition of the BJGP, focuses on four modifiable lifestyle risk factors: smoking, alcohol consumption (excess alcohol intake or never drinking), physical inactivity, and body mass index outside the normal range $(\mathrm{BMl}<18.50$ or BMI >25.00). The researchers demonstrate that women with multiple lifestyle risk factors had higher mortality risks than those reporting none. Assuming causality and reversibility, they estimate that $60 \%$ of deaths of women in this cohort may have been prevented by the avoidance of all four of the modifiable lifestyle risk factors considered.

Examining the study's strengths and limitations, this study is a useful opportunistic exploitation of existing data sets. The weaknesses of utilising this cohort for further studies other than the primary purpose and the conduct of a cross-sectional study within a cohort study have been rehearsed on previous occasions. Similarly the lack of generalisability of the RCGP Oral Contraception Study cohort, which consists largely of white European women who in 1968 were married or living as married, is widely acknowledged.

Relating to this particular study there are issues about the summary classification of low and elevated BMI measurements and the lack of detail of social class (manual or non-manual). The analysis is confined to only four lifestyle risk factors and is based on a single snapshot of lifestyle in middle life, as the researchers were unable to ascertain a full life history of the risk factors. Nonetheless, Iversen et al's recent observations are consistent with those of other UK and US cohort studies of lifestyle factor combinations on mortality. This paper serves as a timely reminder of the relationships between lifestyle and mortality, and re-emphasises the importance of reducing smoking and promoting activity.

While the limitations of the data and methodology of this study will no doubt stimulate debate, more challenging questions emerge as one considers the role of primary care in addressing these epidemiological observations. Iversen et al's study defines and quantifies a problem, but it does not give us solutions to lifestyle modification. Although lifestyle is influenced by a huge range of personal, societal, and environmental factors, general practice has a potentially important role to play, given the $75 \%$ population coverage it provides within a single year. However, as primary care clinicians we still have relatively few robust and effective lifestyle interventions in our armamentarium. So the convention of referring to lifestyle risk factors as 'modifiable' risk factors is misleading as it implies we know how to achieve behaviour change and that there are highly effective interventions ready to be operationalised. Perhaps 'theoretically modifiable' or 'potentially modifiable' would be more realistic terminology?

Within the last 4 years the National Institute for Health and Clinical Excellence (NICE) has published guidance on tackling smoking cessation, ${ }^{3}$ increasing physical activity, ${ }^{4}$ preventing harmful drinking, ${ }^{5}$ and the prevention and management of obesity. ${ }^{6}$ In a recent edition of the BJGP Mercer gave a balanced review of the NICE clinical guidelines on obesity for general practice. ${ }^{7} \mathrm{He}$ highlights deficiencies in the data underpinning the recommendations: little of the evidence is derived from studies focused on a primary care setting, conducted in the UK, or involving patients rather than volunteers. To a greater or lesser degree, Mercer's observations are generalisable to all the NICE guidelines on lifestyle modification.

Further evidence is required to address the deficiencies highlighted above, and future research also needs to address those issues that arise from the complexities of day-to-day general practice. For example, in a consultation with an already overcrowded agenda, how does one most effectively introduce discussion about lifestyle and behaviour change without disenfranchising the patient? What are the best approaches with patients who have repeatedly been unable to sustain lifestyle change? How can the primary care clinician encourage lifestyle change for the patient who already is challenged by coping with everyday life and has severe constraints on their money and time?

Considering the conclusions of Iversen and colleagues' study in the context of 
real patients, competing agendas, and paucity of evidence, one might easily get despondent despite a strong desire to achieve the best for one's patients.

McLandburgh Wilson said 'Twixt the optimist and pessimist the difference is droll: the optimist sees the doughnut, but the pessimist sees the hole'. ${ }^{8}$ We have a professional responsibility to remain optimistic, while seeking robust evidence to inform our primary healthcare practice and our commissioning of interventions to modify patients' lifestyles.

\section{Helen Smith,}

Chair of Primary Care, Brighton \& Sussex

Medical School.

\section{Provenance}

Commissioned; not peer reviewed.

\section{REFERENCES}

1. Royal College of General Practitioners. Oral contraceptives and health. London: Pitman Medical, 1974.

2. Iversen L, Hannaford PC, Lee AJ, et al. Impact of lifestyle in middle-aged women on mortality: evidence from the Royal College of General Practitioners' Oral Contraception Study. Br J Gen Pract 2010; 60: $563-569$.

3. National Institute for Health and Clinical Excellence. Brief interventions and referral for smoking cessation in primary care and other settings. London: NICE, 2006.

4. National Institute for Health and Clinical Excellence. Four commonly used methods to increase physical activity: brief interventions in primary care, exercise referral schemes, pedometers and community-based exercise programmes for walking and cycling. London: NICE, 2006.

5. National Institute for Health and Clinical Excellence. Alcohol-use disorders - preventing the development of hazardous and harmful drinking. London: NICE, 2010.

6. National Institute for Health and Clinical Excellence. Obesity: guidance on the prevention, identification, assessment and management of overweight and obesity in adults and children. London: NICE, 2006.

7. Mercer S. How useful are clinical guidelines for the management of obesity in general practice? $\mathrm{Br} \mathrm{J} \mathrm{Gen}$
Pract 2009; 59(568): 863-868.

8. Wilson M. Optimist and Pessimist. http://www.anvari.org/fortune/Sarcasm/719_twixt-theoptimist-and-pessimist-the-difference-is-droll.html (accessed 12 Jul 2010).

DOI: 10.3399/bjgp10X515034

\section{ADDRESS FOR CORRESPONDENCE}

\section{Helen Smith}

Chair of Primary Care, Room 319,

Mayfield House, University of Brighton,

Falmer, BN1 9PH

E-mail: h.e.smith@bsms.ac.uk

\section{Cardiovascular disease beyond the QOF}

\section{NATIONAL POLICY}

The aim of current Department of Health (DH) policy on cardiovascular disease in England is to improve prevention and treatment in parallel. ${ }^{1}$ The vascular programme within the $\mathrm{DH}$ has made major efforts in recent years to bring prevention efforts together across the whole spectrum of cardiovascular disease, including heart disease, stroke, diabetes, and chronic kidney disease, to combine these with the national clinical directors' work on this programme. In the past, the individual National Service Frameworks ${ }^{1-3}$ have all emphasised the need for prevention, but with slight differences in detail. The current NHS Health Check $^{4}$ programme is evidence of this joined-up approach.

\section{HEALTH AND SOCIAL IMPACT OF CARDIOVASCULAR DISEASE}

Comparing 2005-2007 with 1995-1997, there were, on average, 31000 fewer cardiovascular deaths each year. But there is no room for complacency. Despite the almost $50 \%$ reduction in cardiovascular mortality over the last decade, the reductions have not been equally distributed. There has been a narrowing of the absolute gap in death rates between the spearhead primary care trusts and the English average, and we are on target to reduce the gap by $40 \%$ by 2010, but there remains a major differential between the 1st and 5th quintile of deprivation (Department of Health, unpublished data, 2008). Further improvement will require crossgovernment activity and clinicians need to continue to take into account issues such as deprivation, ethnicity, sex, and age when making clinical decisions with patients and working with them regarding lifestyle and interventions that reduce risk.

\section{IMPACT OF PRIMARY CARE ACTIVITY}

We know from a range of data that primary care has had a substantial impact on cardiovascular burden across the nation where clinical enthusiasm, incentive schemes, and national policies have all played their part. One example is in the prescription of statins where we have seen over a sixfold increase in prescribing, ${ }^{5}$ and a recent comparison of data from 14 developed nations showed that the UK ranks second in terms of utilisation.

The improvement in cholesterol and blood pressure management seen in primary care has been substantial; however, we also know from the Health Survey for England ${ }^{7}$ and cohort studies $^{8}$ that there has been little improvement in the nation's blood pressure, although the percentage of men on treatment has 